\title{
Variations
}

Variations

Revue internationale de théorie critique

$24 \mid 2021$

Echos

\section{Une histoire secrète.}

Du Manifeste communiste à la Théorie critique, ou vice-versa.

\section{Alexander Neumann}

\section{(2) OpenEdition}

Journals

Édition électronique

URL : https://journals.openedition.org/variations/2023

DOI : $10.4000 /$ variations.2023

ISSN : 1968-3960

Éditeur

Les amis de Variations

Référence électronique

Alexander Neumann, « Une histoire secrète. », Variations [En ligne], 24 | 2021, mis en ligne le 03 juillet 2021, consulté le 09 juillet 2021. URL : http://journals.openedition.org/variations/2023 ; DOI : https:// doi.org/10.4000/variations.2023

Ce document a été généré automatiquement le 9 juillet 2021.

Les ami•e•s de Variations 


\section{Une histoire secrète.}

Du Manifeste communiste à la Théorie critique, ou vice-versa.

\section{Alexander Neumann}

L'ouvrage de théorie politique le plus connu et le plus imprimé au monde, Le Manifeste communiste, fait partie du patrimoine mondial de l'humanité. ${ }^{1}$ L'objet est si énorme qu'il convoque tous les hommages, appropriations ou révisions, qu'il incite aussi à tous les abus, détournements, récupérations. La tentative d'actualisation de l'esprit du Manifeste, par Theodor W. Adorno et Max Horkheimer, vient de réapparaitre, exposant une démarche ouverte, subtile et lucide. ${ }^{2}$ Trente ans après la chute soviétique, alors que le socialisme français est un énorme légume chatoyant, la critique du monde tel qu'il va convoque encore les impulsions de la Théorie critique. À chaque époque, il nous faut essayer de reprendre en main la transmission, une nouvelle fois, face au conformisme qui est en passe de s'imposer à elle. ${ }^{3}$ allemande comprend 109 volumes à ce jour ${ }^{4}$ - gardent cette trace écrite d'une prise de position de Marx, à l'encontre du marxisme dogmatique naissant, qui déjà entendait dénaturer sa propre pensée en son nom. Karl Marx affirme en français, après la chute de la Commune de Paris : Si c'est cela le marxisme, je ne suis pas marxiste! ${ }^{5}$

\section{La révolution mondiale}

Le tendre poète Henri Heine, ironique, fantasque mais mordant, formule une belle perspective globale, à l'approche d'un 14 juillet, lorsqu'il partage l'exil de Marx à Paris, en 1842 : la révolution mondiale. La révolution mondiale, la grande lutte de mano à mano des dépossédés avec l'aristocratie des possédants. ${ }^{6}$ Quel contraste, entre l'esprit littéraire et libertaire de ce moment, et les avilissements postérieurs, qui sentent la mort, enterrent le socialisme utopique et le communisme cosmopolite. Ruge et Marx publient à l'époque une revue internationale depuis Paris, les Annales, ${ }^{7}$ qui présente une discussion sous forme de table ronde avec Bakounine, Engels et Heine. Le collectif sollicite Proudhon et les socialistes utopiques, alors que Marx cite George Sand, qui plaide pour un communisme social. Bien entendu, ce n'est pas Lénine, mais Bakounine qui a traduit et publié le Manifeste communiste en russe, en 1869. Communisme peut 
aussi signifier anarchisme, coopération expérimentale, ou féminisme socialiste, dans une vaste mouvance qui prévaut d'ailleurs jusqu'en 1920, sans jamais se dissoudre dans le marxisme. ${ }^{8}$

Les historiens les plus en vue de la bourgeoisie libérale, parisienne, ont introduit un terme assez parlant, pour faciliter la compréhension de ce qui leur était arrivé depuis la grande révolution de 1789 : la lutte des classes. Le concept jaillit dans L'histoire de la révolution française sous la plume de François Mignet en $1814^{9}$, se trouve rapidement repris par le bourgeois versaillais Adolphe Thiers, ennemi de la Commune de Paris. Depuis la révolution de 1789, la noblesse, la bourgeoisie, la paysannerie, les esclaves en outre-mer les travailleurs des villes, peuvent être compris comme des classes sociales, qui entrent tantôt en lutte, tantôt en alliance. Le directeur du doctorat de Marx, le philosophe Edouard Gans, disciple de Hegel, écrit la première histoire allemande de la "révolution française", où la plèbe semble troubler le jeu politique des classes dirigeantes..$^{10}$ Plus nettement, Marx et sa génération vont exposer la lutte des classes comme principe agissant de l'histoire mondiale, écrite du moins.

\section{Le Manifeste}

Le texte n'est pas un livre important, lorsqu'il est tiré à quelques centaines d'exemplaires, à la veille de la révolution internationale de 1848, qu'il précède de quelques semaines. Avec trois mois d'avance, Marx y souligne l'imminence d'une révolution démocratique en Allemagne. Le Manifeste saisit les effets massifs de la révolution industrielle, sa portée mondiale, les grandes différences entre pays mais leur mouvement combiné dans une unité globale, l'importance et la difficulté de l'internationalisme. Travailleurs, unissez-vous, car partout la division l'emporte de fait. L'abolition révolutionnaire de l'esclavage aux Etats Unis, qui suit celui qui s'impose alors définitivement en France, est publiquement soutenue par Marx, qui est aussi l'un des premiers à dénoncer la colonisation de l'Algérie, et le bonapartisme français. ${ }^{11}$ Presque personne ne l'entend, ne l'écoute d'abord, le Manifeste ne sera popularisé qu'à la fin du 19ème siècle en France.

\section{Marx and sons}

Parmi les six enfants de Marx issues de sa vie avec Jenny, née Westphalen, quatre sont des filles, qui ont toutes pour premier nom Jenny, comme leur mère. Jacques Derrida n'a vu que Marx and sons. ${ }^{12}$ Le titre signale l'amputation de toute une part de l'héritage féministe, qui va des hégéliennes de gauche de 1848 comme Louise Dittmar, à Rosa Luxemburg, Clara Zetkin, Emma Goldman, pour se poursuivre chez Angela Davis, ou Nancy Fraser, et plus globalement dans le féminisme socialiste. Bakounine, Marx, Sand, Heine, défendent la liberté sexuelle, et le libre choix des femmes, déjà. Le Manifeste communiste moque ouvertement la panique morale de la presse réactionnaire qui imagine alors que ces communistes veulent imposer le partage des femmes et des enfants.

\section{Marx and friends}

Dans le Manifeste, Engels et Marx commentent l'éventail de la littérature socialiste de leur temps. Ils commencent par rendre un hommage aux écrits de Babeuf, porte-parole du prolétariat révolutionnaire, et de saluer les premiers, véritables systèmes socialistes et communistes: Fourier, Owens, etc. Proudhon est épinglé comme un représentant du socialisme des philanthropes, humanistes et réformateurs bourgeois de toute sorte, 
ceux qui cherchent à atteindre une société dépourvue de contradictions, à éviter la grève ouvrière et la lutte des classes. Le verdict polémique ne doit pas faire oublier le dialogue initial et l'apport de l'imprimeur français, puisque Marx avait souligné que L'oeuvre de Proudhon est un manifeste scientifique du prolétariat français. ${ }^{13}$ Tous deux cherchent alors à s'approprier l'économie politique anglaise. ${ }^{14}$

débat vivant et une lecture disputée des textes. Le marxisme est une invention idéologique qui ne prend corps et se diffuse qu'après la mort de Marx, en 1883. De même, le léninisme est une pure fabrication du régime de Staline, qui s'impose après la mort de Lénine en 1924. Trois mois après, Joseph Staline en personne édicte Les principes du léninisme dans une brochure qui date d'avril 1924. Le marxisme y est défini, paradoxalement, comme une longue déviation opportuniste, depuis la mort de Marx, et l'un des principes du léninisme écrit par Staline est la critique de la spontanéité. A comparer avec le mot d'ordre de Lénine, issu de la révolution des conseils: Tout le pouvoir aux soviets, lorsque l'unité d'action avec les courants socialistes et libertaires prévaut. Si la transformation du vivant en mausolée érige ici une idéologie qui se veut positive, Staline fabrique ensuite deux épouvantails: d'abord le luxemburgisme, contre le mouvement international associé à la mémoire de Rosa Luxemburg, puis à partir de 1928, le trotskysme. Stéréotypes qui peuvent frapper indistinctement toutes les tendances oppositionnelles. Davantage de membres de la direction du parti communiste allemand furent exécutés sous Staline, pendant leur exil à Moscou, que sous la terreur de Hitler. ${ }^{17}$

19 - Avez-vous étudié la Bible avec attention? demande le rabbin.

20 - Non, répond le libre-penseur.

21 - Avez-vous étudié la philosophie juive?

22 - Non, Rabbi.

23 - Alors, dit le rabbin, vous naviguez sous un faux pavillon. Vous n'êtes pas un librepenseur, mais un ignorant. ${ }^{18}$ 

scruter la photo de groupe qui témoigne de son tout premier séminaire de travail, qui a lieu en mai 1923, en Thuringe, au centre de l'action donc. ${ }^{19}$ Car l'Institut für Sozialforschung n'est pas encore officiellement inauguré - raison de l'absence de Horkheimer qui est en train de boucler l'affaire. Friedrich Pollock et Félix Weil sont sur la photo. L'image du séminaire montre en particulier George Lukacs, ministre éphémère de la république des conseils hongroise en 1919, qui assure l'axe de recherche consacré aux études dialectiques, aux côtés de Karl Korsch. ${ }^{20}$ Ces deux auteurs discutent ici en direct des thèses qui sont connues, grâce à leurs livres parues au même moment, dans le cadre de l'axe 2 du séminaire fondateur: études dialectiques. ${ }^{21}$ Comme le directeur désigné, Gerlach, est mort inopinément, l'équipe fondatrice autour de Weil et Horkheimer envisage de confier la direction à Korsch, qui est très pris; il succède à Rosa Luxemburg à la tête du jeune parti communiste, tout en étant professeur de droit à l'Université d'Iéna et ministre de la justice du gouvernement de front unique de Thuringe, en 1923. Les deux principaux animateurs du séminaire fondateur sont donc des ministres issues des révolutions conseillistes, à la pointe du mouvement intellectuel et politique. Beaucoup de femmes chercheuses participent à l'évènement, dont Christiane Sorge et Hedda Korsch, qui ont obtenu des doctorats. Konstantin Zetkin, fils de Clara, rappelle la fondation du 8 mars, journée internationale de lutte des femmes. Richard Sorge, écrivain et journaliste, est également présent, devenu plus tard un sauveur de Moscou, face à l'armée d'Hitler. ${ }^{22}$ Bela Fogarasi et Fukomoto Kazuo élargissent le cercle international.

\section{Tourner la page}

En 1940, Walter Benjamin a souligné le conformisme social-démocrate, sa grande confusion intellectuelle, et le triple problème d'une tradition marxiste qui conduit à la croyance obsessionnelle des politiciens concernés dans le progrès, leur confiance naïve en une base de masse, et leur insertion servile dans des appareils de parti incontrôlés (thèse $\mathrm{X}$ sur le concept d'histoire). ${ }^{23}$ Il est clair qu'il n'épargne pas non plus le marxisme soviétique 
qu'il avait vu de près, à Moscou en 1927. Dès l'après-guerre, en 1950, Karl Korsch élabore dix thèses pour dire toute ce qui ne va pas avec le marxisme dogmatique, dans ses diverses variantes, tandis que Marcuse dissèque le marxisme soviétique. De leur côté, Adorno et Horkheimer s'efforcent d'élaborer les grandes lignes d'une concrétisation politique du programme de la Théorie critique, qui venait de survivre à la guerre sur le plan organisationnel, éditorial, philosophique et sociologique.

\section{Le marxisme soviétique}

Herbert Marcuse, qui avait soutenu la révolution des conseils allemande de 1918 dans sa jeunesse, a réussi à saisir toutes les amputations que produisit le marxisme soviétique, titre d'un de ses livres..$^{24}$ La décision autoritaire de tout miser sur un Etat central, sur la nation et un seul pays, dirigé par un chef, ne pouvait qu'alourdir des apories déjà aperçues lors de la création de l'Union soviétique. Un marxisme soviétique se dresse alors à l'encontre d'un marxisme dit occidental, valorisant de plus en plus des thèmes nationaux et patriotiques. Internationalisme et cosmopolitisme sont relativisés, puis combattues, assimilées à du luxemburgisme. Dans ce discours soviétique, la thèse de doctorat de Karl Marx (1840) se trouve alors brutalement corrigée, car elle souligne la fausse opposition entre idéalisme et matérialisme ${ }^{25}$. Il y voit deux abstractions philosophiques, issues de l'antiquité grecque et de la période d'avant la révolution de 1789, qui doivent être dépassées par une dialectique qui tienne compte de l'expérience historique. A la place, des schèmes binaires s'imposent dans le marxisme, l'opposition entre un matérialisme qui serait marxiste, et un idéalisme qui serait bourgeois sinon réactionnaire. Karl Korsch avait contré cette régression intellectuelle avec la dernière énergie, dans Marxisme et philosophie, après la révolution russe. ${ }^{26}$ De même, le marxisme soviétique postule une opposition schématique entre "base" économique et "superstructures" idéologiques (Marx parle de Grundriss et de Überbau, plan et voute, termes architecturaux complémentaires). Les schémas idéologiques de l'ère soviétique ne se trouvent nulle part dans les 109 volumes qui composent l'oeuvre marxienne.

\section{Coupure, cou coupé}

En 1923, Korsch a insisté sur le fait que la seule orthodoxie intellectuellement acceptable serait la poursuite inlassable d'un travail dialectique. Il met alors en garde contre la tendance du marxisme soviétique de vouloir découper artificiellement des secteurs de la pensée marxienne (et socialiste, communiste ou libertaire). Par endroits, Korsch anticipe déjà les simplifications idéologiques du marxisme soviétique, par exemple lorsque le conseilliste allemand met en garde contre la tentation d'opposer les écrits philosophiques du jeune Marx à l'oeuvre plus accomplie du Capital, une coupure qui signale que ces marxistes n'ont rien compris du tout à la critique de l'économie politique. ${ }^{27}$ Cet avertissement concerne les théorisations ultérieures de Louis Althusser, ou encore de Robert Kurz, qui ont voulu cisailler la pensée critique par des coupures (avant et après le Manifeste de 1848). ${ }^{28}$ Pour Korsch, le marxisme soviétique et son économisme mécanique, hérité de la social-démocratie du 19ème siècle, s'apparente à un travail mort en phase avec la logique d'accumulation du capitalisme, alors que le travail vivant des travailleurs s'exprime par exemple dans l'anarchosyndicalisme ou le mouvement des conseils. ${ }^{29}$ Korsch enfonce le clou, en pointant que les lectures schématiques qui dérivent des interprétations de Lénine, et qui se cristallisent dans un discours léniniste figé après sa mort, retombent in fine à l'état philosophique du débat matérialiste d'avant la révolution française. ${ }^{30}$

\section{Psychologie des masses}



conseilliste de Korsch, à son tour il rend un vibrant hommage à Rosa Luxemburg. Dans son livre de 1922, Lukacs insiste pour sa part sur le fait que toutes les classes sociales baignent dans des fausses représentations, prolétariat et bourgeoisie compris. C'est la raison pour laquelle l'analyse critique doit passer, pour Lukacs, par l'élaboration d'une typologie sociologique (Typologie), et par la compréhension de la psychologie des masses (Massenpsychologie, terme introduit par Freud à cette époque). ${ }^{31}$ Cette orientation est très proche du programme d'enquête original de l'Institut de Francfort, qui va être déployé en exil aux USA. ${ }^{32}$ Lukacs, lui, n'aura pas l'occasion d'y participer, se sentant contraint de renoncer aux études dialectiques, pour développer un discours d'abord conforme aux desiderata du régime de Staline, pendant son exil à Moscou (à partir de 1933). C'est ici que le fil se rompt.

41

\section{Destruction et renaissance}

Herbert Marcuse fait le point après la mort de Staline. Tout comme il a fait tuer pratiquement tous ses opposants, sa théorisation générale, imposée, a détruit plus profondément la dialectique que n'importe quelle révision. ${ }^{33}$ Alors qu'au moment du Manifeste communiste, la pensée dialectique cherche à saisir le mouvement conflictuel de la lutte des classes (qui existe empiriquement en dehors de la théorie de Marx), dans une compréhension des antagonismes, la théorie stalinienne réprime cette pensée, en inventant l'idée de contradictions non-antagoniques, puisqu'il ne faut pas admettre des luttes et désaccords en Union soviétique. Staline fait supprimer très officiellement la conception dialectique de la négation (ou négation de la négation), dès 1938. Cet appareillage répressif se trouve ensuite chez Mao, Althusser et une série d'auteurs marxistes-léninistes. Le livre d'Adorno, Dialectique négative, est une vive réfutation de cette réification idéologique. ${ }^{34}$ Dans des notes peu connues, Horkheimer montre dès 1931 comment Lénine s'écarte déjà du travail dialectique, dans sa lutte idéologique contre des adversaires socialistes, préférant la proclamation de principes dogmatiques, de facture métaphysique, à une analyse concrète des conditions historiques et sociales dans lesquelles naissent les théories conventionnelles. ${ }^{35}$ Marcuse se pose ensuite la question de savoir, dans quelle mesure les écrits de Lénine peuvent entrer en conflit avec le marxisme soviétique, sans trancher définitivement la discussion.

\section{Héritage}

La pensée dialectique est la tentative de rompre le caractère contraignant de la logique par le recours aux moyens de la logique. Mais ... elle s'expose sans cesse au danger de succomber à son tour à cette imposition contrainte: la ruse de la raison cherche à prendre le dessus, même à l'endroit de la dialectique. ${ }^{36}$

\section{Pour un nouveau Manifeste}

Après la mort de Staline, Horkheimer et Adorno veulent se souvenir de la période qui a fait naitre les premiers mouvements socialistes en Europe, avant 1848, lorsque ces mouvements expérimentaux ne sont pas identifiés à des Etats, des bureaucraties, avec leurs idéologies refroidies. Au printemps 1956, ils préparent la rédaction d'un nouveau Manifeste, dialogue rendue possible et mis en forme par la docteure Gretel Adorno, un manifeste qui ne soit pas entravé par le marxisme soviétique et sa ramification maoïste, mais capable de relancer les ressources critiques des révolutions conseillistes d'avant 1923. Le leitmotiv de leur discussion est la relation entre théorie et pratique, qui forme le titre allemand de ce texte. ${ }^{37}$ Pour Adorno, cette approche doit être plus radicale que le 
léninisme, qui s'est retournée en production idéologique, mais il envisage une rédaction léniniste, dans son intention révolutionnaire et internationaliste, susceptible de subvertir les partis du communisme doctrinaire qui ont pour base arrière la Russie et la Chine. Horkheimer y voit un écho de Trotsky, ce qui n'est pas exact, car Adorno ne prétend pas que l'Etat soviétique puisse être sauvé. Il propose aussi de faire exploser la jonction entre le marxisme étatiste et l'ontologie philosophique, cette fois en accord avec Horkheimer. Dans ce dessein, Adorno cherche les moyens pour déborder en pratique les sentiments populaires vagues en faveur du socialisme, et de provoquer les institutions bourgeoises à l'Ouest en ciblant les failles du discours établi, dont "le tabou sexuel". Max Horkheimer ne cesse de rappeler à son ami, à plusieurs reprises, qu'il défend là des arguments qui rejoignent la position de Herbert Marcuse (Attention, Marcuse!). Adorno pense que le monde administré, cette conjugaison de la bureaucratisation et du capitalisme global auquel le modèle soviétique n'échappe pas plus que le fordisme américain, ne pourra pas trouver une stabilité durable. La persistance de la spontanéité dans les masses enrôlées est trop forte et va resurgir tôt ou tard, insiste-t-il, en 1956. Une nouvelle révolution des conseils va éclater la même année, en Hongrie, au mois d'octobre, quelques mois après la discussion de Horkheimer et Adorno à propos d'un nouveau Manifeste.

Attention, Marcuse!

Adorno juge que les citoyens russes et adhérents au parti soviétique ne supportent la direction soviétique, après Staline, qu'à force de recevoir des "coups sur la tête". Le repli sur l'autorité n'est qu'un pis-aller, face aux humiliations subies en permanence, à l'Ouest aussi. "C'est exactement la position de Herbert Marcuse", remarque Horkheimer, là encore. Marcuse pensait que le stalinisme fonctionnait comme un épouvantail et un blocage collectif; son affaiblissement devait libérer des énergies dans les années 1960. Il est vrai qu'Adorno va rejoindre ses positions, comme il le signale dans une dernière lettre à Marcuse en 1969. Les universitaires qui espèrent trouver une forme de modération antimarxiste chez Horkheimer seront dépités, à la lecture attentive du texte qui l'unit à son ami Adorno. Car pour Horkheimer, il s'agit ici de démontrer que les démocrates et la social-démocratie ont trahi l'humanité tout entière, trahissant autant l'impulsion première des Lumières que la conception initiale de la dictature $d u$ prolétariat. En pratique, Horkheimer souligne que la critique doit montrer qu'il ne se passera rien, tant que des groupes agissants ne font rien pour que le changement historique se produise, ce qui implique d'oeuvrer en faveur d'une scission globale dans le mouvement communiste international.

\section{Chaise musicale}

Le problème cardinal que soulève Horkheimer ici est que l'espace qui correspond à leur intervention politique et critique, vacant à l'époque de Marx et Proudhon, est désormais occupé par des partis communistes de masse. Contrairement à 1848 ou 1917-18, cela provoque le problème nouveau que ces partis transforment toute discussion politique en réification idéologique, alors que pour être compris par le public large qu'ils influencent, il faudrait se rapprocher de l'argot marxiste en vigueur, au détriment de la critique. De plus, pareil Manifeste va être interdit de circulation en Russie et en Chine, marginalisé en Amérique et en Europe de l'Ouest, à l'exception notable de la France et de l'Italie sans doute, précise Horkheimer. Adorno reconnait qu'une mouvance socialiste autonome serait rapidement polarisée, en 1956, entre communistes dominants et mainstream social-démocrate. Il faudrait éviter que cela ne 
serve de prétexte à une grande alliance rhétorique sans contenu, qui irait de la socialdémocratie allemande à la Chine populaire. Plus récemment, Andrew Feenberg a montré qu'il n'a rien saisi de cet avertissement, en se moquant de cette réflexion pour mettre à sa place une "alternative réaliste" pour une "gauche progressiste", indéterminée, incluant la position de Habermas, à la place d'une analyse politique concrète, critique..$^{38}$

\section{Antinomie}

Le dilemme politique que soulignent les Francfortois désigne une antinomie, d'un point de vue théorique. Adorno la résume : d'une part, la théorie doit maintenir la possibilité de penser comment le communisme peut s'introduire concrètement dans des rapports de pouvoir précis, mais de l'autre, cette même théorie va aussitôt subir la pression décuplée de choisir parmi des fausses alternatives. Ces fausses alternatives sont facilement repérables dans le texte : marxisme soviétique ou néocapitalisme; pseudoactivité partidaire ou abstention politique; art idéologiquement engagé ou art apolitique; antihumanisme ontologique ou humanisme bourgeois, abstrait et désincarné. La pression en faveur de la fausse alternative favorise le non-sens, précise Adorno. Horkheimer esquisse une position de repli, qui consiste à maintenir l'exigence pratique pour le changement en évitant de dire aux gens ce qu'il faut faire, alors qu'Adorno trouve qu'il s'agit de rechercher une unité tendue, entre théorie et pratique. Il pense qu'une idée critique juste pourra toujours se réaliser à l'avenir, à condition de sortir du marxisme. Les corédacteurs francfortois tombent d'accord pour relancer le riche héritage des socialistes utopiques qui est déjà discuté dans le Manifeste de1848 : Proudhon, Fourrier, Owen. Adorno glisse que ce socialisme n'est pas tellement utopique, mais reste pratique, au fond.$^{39}$ La perspective utopique apparait séduisante, dans un moment qui semble presque désespérant pour Horkheimer, alors que Adorno marque au confiance dans une relance critique proche, précisément parce que les rapports de domination se montrent dans toute leur évidence. La formulation concise, incisive, de la critique, même si elle a peu de chances de s'incarner dans des mouvements oppositionnels à une grande échelle, en 1956, peut du moins servir de lettre qui voyage dans une bouteille jetée à la mer, une Flaschenpost.

\section{L'idéologie allemande, en salade}

Le marxisme soviétique, puis les déclinaisons de la doctrine de Staline de 1938, on voulu couper l'idéologie marxiste des écrits de Marx, dont la lecture directe reste potentiellement subversive et anti-dogmatique. Cette ligne idéologique passe par la découpe de l'oeuvre marxienne en deux, avant et après le Manifeste communiste de 1848. Les textes de Marx furent donc tronqués et réassemblés en fonction d'une idéologie d'Etat qui s'est propagée à travers l'espace soviétique mondial (Europe, Asie, Afrique). Il est aujourd'hui documenté, à l'aune de l'édition scientifique allemande, que Marx n'a jamais conçu de livre appelé L'idéologie allemande, titre fabriqué par les éditions soviétiques sous Staline en 1932, après l'élimination du directeur-fondateur Rjazanov en 1931, et qui ont composé cette publication à partir de divers manuscrits de plusieurs auteurs associés, comme bon leur semblait. ${ }^{40} \mathrm{~L}^{\prime}$ intention semble claire: présenter les textes de Marx en conformité avec un prétendu marxisme, qui écarte l'émancipation des travailleurs par eux-mêmes, la critique de l'Etat et tout internationalisme. C'est le sens d'un rejet en bloc de l'idéalisme, de l'utopisme socialiste, du cosmopolitisme, d'un élan révolutionnaire polyphone et incontrôlé, du féminisme conçu et pratiqué par les 
femmes. Les auteurs proches de Marx, ou en dialogue critique avec lui, doivent ici s'effacer, Blanqui, Bakounine, Owen, Proudhon, Sand, Heine et alli.

\section{L'axe Francfort-Moscou}

En réalité, le projet d'éditer les écrits de Marx, Engels et associés de manière intégrale, est directement lié à la création de l'Institut de Francfort en 1922-23. De même que la Théorie critique s'amorce sur fond des révolutions conseillistes, le projet d'éditer les écrits de Marx de manière complète émerge lors de la création de l'Institut de Francfort, en 1922-23. Aujourd'hui, tout le monde peut savoir que l'équipe fondatrice de l'Institut für Sozialforschung, avec Félix Weil et Carl Grünberg, a organisé la première édition scientifique des écrits de Marx et Engels, en coopération avec David Rjazanov, directeur de l'Institut Marx-Engels à Moscou, pendant que Horkheimer organise l'Institut. ${ }^{41}$ La coopération est devenue de plus en plus difficile, à mesure que la pression stalinienne s'est accrue, pour se rompre définitivement en 1928. Ecarté, Rjazanvo fut finalement exécuté, au terme d'un procès politique. Nota bene: le marxisme soviétique n'a pas permis la diffusion des arguments de Marx, sous Staline, mais l'a entravé. Bakounine, Weil et Rjzanov sont les références éditoriales.

\section{Le Capital en 13 volumes}

Aujourd'hui, l'édition MEGA des oeuvres de Marx et Engels présente le Capital en 15 volumes, dont deux volumes de traduction vers les langues française et anglaise. ${ }^{42} 13$ volumes restituent ainsi les travaux et manuscrits allemands de Marx qui concernent $L e$ Capital, ou plus précisément la critique de l'économie politique. Comme la présentation est chronologique, il est possible de voir comment les manuscrits et esquisse de 1844 sur la critique de l'économie (vol.3 et 4) préparent la rédaction du Manifeste communiste (vol.6), pour se prolonger dans les premières esquisses de la critique de l'économie politique de 1857, les manuscrits de 1963, et les textes composant Le Capital (II, vol. 1-15). Rien ne suggère un abandon de la pensée dialectique, présente au moment du Manifeste communiste, en faveur d'un développement de l'être sans antagonismes, qui serait donc ontologique. C'est là une piste qui se trouve chez Althusser et le Lukacs tardif. Vérification faite, la conception ontologique du temps, les mots ontologie ou ontologique, n'apparaissent pas une seule fois, dans les 13 volumes des écrits de Marx dont il s'agit.

\section{Legs}

Aucune génération n'a encore pu discuter l'étendue des 109 volumes actuels dans le détail et dans son ensemble, en partant des nombreuses contradictions et paradoxes de l'oeuvre marxienne, qui furent déjà relevés par des esprits brillants, par exemple Rosa Luxemburg. Elle avait pointé, dans L'accumulation du Capital, que le calcul précis des sommes, de l'accumulation financière au cours des étapes du circuit économique, fait apparaitre un important écart avec le modèle théorique imaginé par Marx ${ }^{43}$ Elle en a déduit que les conflits économiques entre puissances capitalistes, à l'échelle mondiale, devaient logiquement s'aiguiser, puisque les capitaux financiers ne trouveront pas de débouché à terme, en 1913. Cette clairvoyance permit de saisir la première guerre mondiale, et la possibilité de la révolution russe.

\section{Lire le Capital}

En accord avec les arguments qu'il a exprimé pour un nouveau manifeste, Adorno se focalise sur la formation d'une nouvelle génération critique à Francfort. Dans ce contexte, il soutient la création d'un groupe d'étude sur les Grundrisse de Marx et sa 
critique de l'économie politique, à l'initiative de Hartmut Reichelt, en 1962. Cela s'inscrit dans une continuité avec l'édition des oeuvres de Marx par l'Institut de Francfort depuis 1922-23. Ici réside la source première. Les cours de philosophie d'Adorno, qui abordent Marx, sont alors très prisés et fréquentés. Y participeront tour à tour Oskar Negt, Angela Davis, Heide Berndt, Gerhard Steinert, Elisabeth Lenk, Regine Becker Schmidt - devenus professeurs d'université par la suite - ou encore Jürgen Krahl. ${ }^{44}$ Habermas, lui, avait alors déjà quitté le courant critique pour se rapprocher du platonicien Hans Gadamer (avant de se détourner publiquement de la Théorie critique). Hans Jürgen Backhaus, qui a restitué ses notes d'un cours adornien sur Marx, y participe aussi, avant de se faire connaitre comme disciple de Reichelt au sujet de la nouvelle réception marxienne, Neue Marx Lektüre. ${ }^{45}$

\section{L'abstraction réelle}

$\mathrm{Au}$ cours de l'approfondissement de ses propres recherches marxiennes, Adorno va alors rencontrer Alfred Sohn-Rethel, qui avait cherché à s'intégrer dans l'Institut de Francfort dans l'entre-deux-guerres. En 1965, les deux théoriciens discutent et précisent la question de l'abstraction de l'échange, ou des abstractions réelles qui découlent du fétichisme de la marchandise sous le capitalisme. ${ }^{46}$ Adorno y retrace finement la correspondance entre le mouvement de la lutte des classes et l'apparition des catégories philosophiques de l'échange. Sohn-Rethel, rejoint par Adorno, a relevé l'erreur qui consiste à voir l'échange comme un acte dénué de pratique, par opposition à la production d'objets d'usage, préférant voir deux types de concepts et de pratiques, qui entrent en conflit de manière dialectique et nourrissent la critique. Les fausses représentations, fétichistes, ne sont pas moins des formes mentales objectives. Le rapport marchand dans le capitalisme est une abstraction, mais cette abstraction est réelle et non pas factice, car elle engendre des rapports sociaux spécifiques, qui constituent la société. Backhaus a concrétisé cette impulsion, Jean-Marie Vincent l'a reçue, transmise et transposée dans l'espace francophone. ${ }^{47}$ Marx après les marxismes devint ainsi son leitmotiv, sa ligne éditoriale. ${ }^{48}$

\section{Capitalisme tardif}

Les réflexions libres sur Marx trouvent une expression éclatante au congrès de sociologie de Francfort de 1968, qu'Adorno préside, dans son introduction sur le capitalisme tardif. ${ }^{49} \mathrm{Il}$ s'agit alors de promouvoir l'héritage conceptuel et critique de Marx, à l'encontre du marxisme réifié, du positivisme et du libéralisme. Ce sera une sorte de testament, à côté de sa Dialectique négative. Bien entendu, le développement dialectique qu'il y expose est en tous point en phase avec sa réception marxienne : L'exigence d'une unité de la pratique et de la théorie a irrésistiblement asservi la théorie; la dépouillant de tout ce qu'elle aurait pu apporter à cette unité..$^{50}$

\section{Affinités}

A la même époque où le penseurs de Francfort discutent les Grundrisse, la discussion reprend aussi dans d'autres pays, dans le monde anglophone, avec la Left review, en Italie avec les Quaderni rossi et Mario Tronti en particulier, ou à Paris avec Guy Debord qui connait un développement solitaire. Ici, la revue Socialisme ou barbarie avait déjà défriché le terrain, avant 1968, à côté du cercle de Pierre Naville, fondateur de la sociologie au CNRS depuis la Libération..$^{51}$ Dans ces sillons, Miguel Abensour ou JeanMarie Vincent vont reprendre la promotion des apports de la Théorie critique après 1968, en France. La liste des auteurs venus étoffer le débat est trop longue pour être déroulée ici, elle inclut Albert Einstein. ${ }^{52}$ Dernière venue dans ces efforts de 
compréhension, l'école althussérienne avait peiné à rattraper l'ample discussion, elle va d'abord transposer les leitmotivs du marxisme soviétique dans les termes du débat français, sans citer aucune autre approche.

\section{Certes}

L'école althussérienne qui a produit le livre collectif Lire le Capital en 1965 assure dès la première page: Certes, nous avons tous lu le Capital. Pourtant, Althusser affirme, à la fin de sa vie, qu'il n'avait alors presque rien lu, excepté le tout premier volume du Capital et quelques articles philosophiques en français. ${ }^{53}$ En 1965, il postule une coupure de l'oeuvre marxienne, qui sépare l'avant de l'après 1848, année du Manifeste et de la révolution - Althusser coupe ainsi ce texte en deux, dans une forme de découpe. Pour Marx (1965) cite Staline et son modèle de contradictions non-antagoniques. Dans L'avenir dure longtemps, Althusser a donné à voir à quel point il avait adhéré aux schémas du marxisme soviétique, allant jusqu'à justifier le pacte Hitler-Staline au passage. ${ }^{54} \mathrm{Il}$ expose aussi pourquoi il n'a pas participé aux mouvements majeurs de son temps, à la lutte des classes: la grève générale de 1936, la Libération, la révolution algérienne (1954-62), la grève générale de Mai 68, ou l'élan antibureaucratique qui produit la chute du mur de Berlin en 1989.

\section{Leçon, lésion ou illusion d'Althusser}

Les apories althussériennes, le caractère abstrait et dogmatique de son discours, ont été amplement exposées de son vivant, en particulier par l'ancien disciple althussérien qu'est Jacques Rancière (La leçon d'Althusser), par l'intellectuel communiste Lucien Sève, par l'historien E.P Thompson (Misère de la théorie), par Jean-Marie Vincent (Contre Althusser), avec Catherine Colliot-Thélène, Denise Avenat, Alain Brossat, Daniel Bensäid, Ernest Mandel, puis Cornelius Castoriadis (Les crises d'Althusser), Oskar Negt et Alexander Kluge, tous deux formés par Adorno (Histoire et subjectivité rebelle). ${ }^{55}$ Guy Debord se contenta de parler du sombre déni qui caractérise l'attitude althussérienne envers le concept du fétichisme de la marchandise. ${ }^{56}$ Claude Sarraute a pointé le problème de l'impunité du féminicide, le meurtre d'Hélène Rytmann. La revue Actuel Marx vient d'intituler tout un numéro : ALTHUSSER. ${ }^{57}$ Si l'idée d'honorer les auteurs historiques est excellente, le fait demeure que ce numéro n'aborde aucun des actes et prises de positions problématiques qu'Althusser a voulu exposer lui-même, et omet d'évaluer les critiques qui lui ont été adressées depuis un demi-siècle. En réalité, l'éclatement du collectif althussérien de 1965 a libéré, de manière centrifuge, contradictoire, caléidoscopique, diverses approches, abandons ou élaborations originales. $^{58}$

\section{They, the people}

Le fait que les intellectuels fréquentent le plus souvent des intellectuels ne devrait pas les pousser à se considérer comme des personnes plus mesquines que le reste de l'humanité... Une grande partie de l'anti-intellectualisme et de l'irrationalisme, qui monte jusqu'à Huxley, est actionnée par le fait que les auteurs se plaignent du mécanisme de la concurrence, sans le percer à jour, ce qui fait qu'ils lui succombent... Pour cette raison, ils fréquentent à la fin les temples indiens. ${ }^{59}$

\section{Contradiction mortelle}

Le capitalisme reste plein de contradictions, potentiellement explosives, comme le montre les crises économiques récentes. Le Manifeste communiste le voit confronté à une seule contradiction, mortelle, entre forces productives et rapports de distribution, entre prolétariat et bourgeoise. L'idée centrale, qui reste à prouver, se réverbère dans la 
construction du Capital, mais ne trouve pas de cohérence théorique globale. Marx calcule le degré d'exploitation, la plus value absolue et relative, sur la base d'une production donnée de biens, de vêtements par exemple. Cela concerne un nombre de salariés qui participent à cette production précise. Mais plus loin, Marx explique que cette plus value ne se transforme en profit qu'à travers le marché, qui tend à se mondialiser. A cette échelle, celle du marché qui réalise l'abstraction nourrissant le capital, son calcul initial d'une production de vêtements n'est plus exacte, sinon fausse, la logique change. L'auteur du Capital ajoute lui-même que la valeur potentielle peut ici se perdre (être dévaluée ou détruite par des crises), que le crédit, les banques, la bourse, le financement différé sont une condition nécessaire à la production capitaliste. Le tout se passe alors à un autre niveau que le calcul initial de la plus value. ${ }^{60}$ De même, la marchandisation globale du travail salarié, sa soumission réelle au marché, implique que sa réalité n'est plus circonscrite à la seule production, mais implique la logistique, les transports, la communication. ${ }^{61}$ Le travail concret et le travail socialisé suivent des logiques propres. Marx signale encore que la valeur de la force de travail comporte un élément moral et un élément historique, qui échappe au calcul de base. ${ }^{62}$ Certes, l'exploitation, les contradictions et crises demeurent, mais Le Capital ne connait pas leur dénouement. La contradiction n'est pas mortelle, mais terrible. Les postulats qui veulent croire que la production s'oppose en soi à la finance, ou que tout va s'effondrer, sont des émanations tardives d'une vulgate marxiste, qui ne cherche pas à comprendre.

\section{Que faire?}

La question de savoir que faire, est intuitive, elle a été formulée de manière nette par Kant, au moment de la révolution de $1789 .{ }^{63}$ Le philosophe y examine la relation entre la théorie et de la pratique. Plus tard, Lénine postule une unité de la théorie et de la pratique, sans l'assurer. Le que faire de Lénine de 1902 se réfère à une avant-garde, mais qui ne devrait exister qu'en rapport à un mouvement large, qui décide lui-même de la direction à suivre. En théorie, tout le pouvoir doit aller aux conseils, mais il décide ensuite de dissoudre de force l'assemblée constituante qui a été convoquée par les conseils, en janvier 1918. Incohérence et pragmatisme sans concept, qui ouvre la voie à l'abus. ${ }^{64}$ En 1956, Horkheimer et Adorno savent qu'il ne faut sous aucun prétexte répéter l'erreur fatale. Que faire? Maintenir la critique, former une jeune génération, encourager les mouvements spontanés, oppositionnels, internationaux.

\section{Association socialiste}

L'héritage du Manifeste, des écrits de Marx se de sa génération, de la révolution des conseils, sont les sources documentées de la Théorie critique. Ce développement rebondit en 1968. Le rôle de Marcuse dans l'émergence d'une nouvelle gauche internationale est clair, moins connu est la contribution francfortoise à l'émergence du premier courant significatif à gauche du parti social-démocrate en Allemagne de l'Ouest, alors que le PC n'y jouait qu'un rôle marginal. L'apparition d'un mouvement anti-autoritaire dans la jeune génération, dans les années 1960, provoque alors l'expulsion de l'organisation étudiante du SPD, le SDS. Le jeune mouvement est d'abord proche de l'opposition de gauche du SPD, dont l'un des porte-paroles est Wolfgang Abendroth, professeur en science politique qui présenta une motion minoritaire au congrès de Bad Godesberg de 1959 (en phase avec la révolution française, la critique du capitalisme et contre la restauration). Le parti décide ensuite former un gouvernement avec la droite traditionnelle. Abendroth participe à la création d'une association de soutien au mouvement étudiant socialiste, après l'expulsion, et à la quelle appartient 
Gerhard Brandt, le successeur direct d'Adorno à la direction de l'Institut de Francfort en 1969. Oskar Negt et Jürgen Krahl - dont Adorno a dirigé les thèses - servent d'intermédiaire entre le SDS, les professeurs socialistes, et les syndicats. ${ }^{65}$ Horkheimer et Adorno répondent à l'invitation du SDS pour donner des conférences, leurs écrits font partie du matériel de formation, à côté de Marx, Luxemburg, Lénine, Korsch et Marcuse. Lors de la conférence du SDS de 1962, Elisabeth Lenk, porte-parole de la Théorie critique francfortoise, parvient à convaincre mille délégués présents de maintenir cette exigence intellectuelle, au lieu de partir dans un activisme sans direction précise. Et lors d'une conférence en défense du Vietnam du SDS en 1966, contre l'empire, 2200 personnes viennent écouter Abendroth, Negt, et Marcuse. ${ }^{66}$

\section{Etat d'urgence}

Suite à des attentats à la bombe dans un grand magasin, commis par Hans Baader et Ulrike Meinhoff, le gouvernement Kiesinger annonce l'adoption d'une loi en faveur de l'état d'urgence. Adorno saisit parfaitement que l'Etat autoritaire, sans être lui même fasciste, peut bel et bien conduire au fascisme, et c'est justement l'article constitutionnel 48 de la république de Weimar concernant l'état d'exception qui avait permis l'accès au pouvoir des nazis sous Hitler. Adorno décide d'agir, en accord avec l'Institut de Francfort. Son texte de 1968 : Gegen die Notstandsgesetze - Contre l'état d'urgence - est le témoignage le plus clair de son engagement public. Il y souligne la manoeuvre qui avait permis la prise de pouvoir des nazis dans le passé, avant de commenter de manière sarcastique les errements des gouvernements de droite depuis l'après-guerre. Les tendances qui penchent en faveur d'une restauration politique autrement dit vers la contre-révolution - s'accroissent, constate-t-il : "Seul un vilain optimisme peut voir autre chose dans ces lois pour l'état d'urgence qu'un renforcement de ces tendances" ${ }^{67}$ Dans la perspective de lancer une campagne publique visant à empêcher l'adoption des lois permettant l'état d'urgence, Adorno s'associe à son ancien doctorant Negt, qui est l'un des responsables du SDS. Ensemble, ils parviennent à convaincre Otto Brenner, le dirigeant du syndicat de la métallurgie, la première fédération syndicale de l'Europe, de s'unir contre l'état d'urgence. ${ }^{68} \mathrm{Krahl}$ prend la parole lors d'une manifestation dans la capitale qui réunit 60 mille personnes. La coalition des intellectuels critiques, des jeunesses socialistes et des syndicats marqués par l'antifascisme parvient à exposer le caractère problématique de cette voie, et d'en limiter l'usage. Serait-ce un modèle critique pour la France contemporaine? Comment se fait-il que des marxistes dogmatiques continuent à prétendre qu'Adorno aurait soutenu l'Etat autoritaire et la police?

\section{Occupé}

Par la suite, la radicalisation d'une partie des étudiants aboutit à l'occupation de l'Institut de Francfort, en 1968. Adorno discute longuement avec les occupants, qui finissent alors par partir volontairement.$^{69}$ Une légende urbaine prétend qu'une intervention violente de la police aurait mis fin à cette action, ce qui est faux. L'action fait réfléchir Adorno, en phase avec Marcuse sur ce sujet, comme le montre leur correspondance. Marcuse à Adorno: Ce que tu dis au sujet de l'évolution du mouvement étudiant se recoupe complètement avec mon expérience: des éléments rationnels et irrationnels, sinon contre-révolutionnaires s'y mêlent inextricablement..$^{70}$ Début 1969 , une seconde occupation, destinée à préparer une action contre l'Ambassade d'Espagne, provoque un dépôt de plainte de la direction qui craint une sanction publique, et en ce sens une implication de la police, qui interroge alors le porte-parole déclaré, Krahl. L'évènement 
stimule une vaste propagande marxiste-léniniste contre la Théorie critique, qui parle de professeurs indics et supporteurs de flics, dans un mouvement qui va aussi nourrir la future RAF, Fraction de l'armée rouge. Ulrike Meinhoff, qui avait écouté un cours d'Adorno, était d'abord rédactrice du magazine konkret, désigné comme une publication néostalinienne par le SDS, avant de lancer la RAF, puis de se suicider en prison. ${ }^{71}$ Adorno meurt en 1969, Krahl en 1971, et l'association initiale entre mouvements contestataires et Théorie critique se disperse un temps, sous l'effet de la restauration étatique, et des actions commandos de la RAF. Cette organisation s'est auto-dissoute en 1998, son échec se lit dans un banal communiqué. ${ }^{72}$ Adorno et Marcuse, auraient-ils subodoré cette fin sans perspective? FBI, Angela Davis se trouve ensuite emprisonnée. Marcuse, Abendroth et Negt organisent alors de grandes manifestations pour sa libération, aux USA et à Francfort, en 1972. Davis est une étudiante de Marcuse, qui sur son conseil avait étudié chez Adorno et Negt à Francfort en 1965, et qui leur rend toujours hommage. Elle incarne ainsi une forme de créolisation de la Théorie critique internationale, après avoir examiné la philosophie politique allemande, depuis Kant, Hegel et Marx. ${ }^{75}$ L'un des textes de Marcuse qui facilite leur rencontre porte un titre d'inspiration kantienne, Critique de la tolérance répressive. Lors de son séjour en prison, elle entame l'écriture de son livre Femmes, race et classe, qui choisit 1848 comme point de départ d'une compréhension de la lutte des classes aux Etats Unis. ${ }^{76}$ L'esclavage, condition de classe, sa transformation en salariat subalterne, le mouvement de libération des femmes, noires subalternes ou petite-bourgeoises (pour ainsi dire blanches à cette époque), sont ici comprises comme des relations et alliances de classe dynamiques, contradictoires et changeantes. La théorie de l'intersectionnalité semble complètement ignorer cet apport, issu de la Théorie critique. Plus récemment, Nancy Fraser, Tithi Bhattacharia et Cinzia Arruz ont repris l'héritage critique international, en se référant explicitement au modèle initial du Manifeste communiste de 1848 et à l'orientation de Davis. ${ }^{77}$ 

ontologique, en dehors de la Théorie critique. Dans sa récente histoire de la philosophie en 2 tomes, ce développement s'arrête juste avant le Manifeste communiste. ${ }^{78} \mathrm{~S}^{\prime} \mathrm{il}$ n'y a pas unité entre théorie et pratique, reste-t-il du moins une relation, une perspective, pour cet intellectuel politique? Oui : Macron présente trois traits caractéristiques sortant des cadres habituels: son courage à façonner une politique; sa conscience aiguë de la nécessité de transformer le projet européen, jusqu'ici porté par les seules élites, et d'en faire un projet porté par les citoyens, fondé sur une auto-législation démocratique des citoyens; et le fait qu'il croit au poids des mots (...) Par son ambition de concevoir en termes politiques les problèmes d'une société mondiale de plus en plus interdépendante, Macron se démarque de ces bureaucrates de la politique chroniquement dépassés par les événements et y réagissant au jour le jour, avec opportunisme, incapables de proposer la moindre perspective. (...) Rarement une ascension aussi inattendue que celle de cette personnalité fascinante, peut-être éblouissante, en tout cas exceptionnelle, aura témoigné à ce point des contingences de l'histoire. ${ }^{79}$

Le souvenir de 1848, celui des révolutions conseillistes de 1917-23 et de leur élan intellectuel se fait sentir à nouveau, aujourd'hui, à mesure que de grands mouvements démocratiques et insurrectionnels jaillissent aux quatre coins du globe, ces dix dernières années. Il y a un siècle, l'élan de la révolution des conseils allait spontanément vers la convocation d'assemblées constituantes, en Russie, et en Allemagne. La Tunisie, l'Islande d'abord, puis le Chili ont emprunté cette voie, sous l'effet d'immenses soulèvements. Le Chili, cas en apparence désespéré, renverse l'héritage dictatorial, décide écrire une constitution égalitaire. La constituante élue en 2021 est composée de tous les courants égalitaires, libertaires, démocratiques, féministes ou encore communistes possibles, dont les prémisses apparaissent dans le Manifeste de 1848. Angela Davis, au Chili, en 2016 : Je n'aime pas être une icone, mais je me rends compte que la circulation de mon image encourage un pouvoir populaire, j'espère que ce mouvement se souviendra, qu'à l'encontre de défis insurmontables, face aux personnages les plus puissants au monde, nous avions fini par gagner. ${ }^{80}$

\section{Rouge à lèvres}

Plus discrètement, la réception de la Théorie critique, planétaire et créolisée, a pris un rythme plus soutenu dans la dernière période. Le débat intellectuel est encore frêle, le doute permis. Dans une nouvelle génération étudiante et salariée, anglophone, hispanophone, francophone, polyphone, l'approche de la Théorie critique trouve une oreille ouverte, se développe dans de nouvelles conditions. La Théorie critique n'habite plus à Francfort, mais à New York, Hanovre, Mexico City, San Cristobal, Seoul, Sao Paolo, Dakar, Los Angeles, Santiago, Buenos Aires, Paris aussi. Tout comme le centre expérimental de Vincennes ne réside plus à Vincennes. Greil Marcus a imaginé que la Théorie critique puisse faire partie d'une histoire secrète du vingtième siècle. ${ }^{81} \mathrm{Il}$ est possible qu'elle soit elle-même l'histoire secrète qui mène de la révolution conseilliste, qui fit débuter le 20ème siècle, à l'ouverture d'un nouveau siècle qui vient à peine de s'ouvrir. 


\section{NOTES}

1. K.Marx, F.Engels, Le Manifeste communiste, 1848:

http://classiques.uqac.ca/classiques/Engels_Marx/manifeste_communiste/

Manifeste_communiste.pdf

2. Adorno/Horkheimer, Vers un nouveau manifeste, éd. de la tempête, 2020.

3. W. Benjamin, Sur le concept d'histoire, thèse VI (nous traduisons : Benjamin, Über den Begriff der Geschichte in : Sprache und Geschichte, Reclam, 2000, p.144).

4. Voir mega.bbaw.de

5. K. Marx, cité d'après F.Engels, en français dans le texte, MEW 37, Dietz, p.463.

6. H.Heine, Lutetia, XLVI, Paris, 12 juillet 1842, Reclam, 1920 (nous traduisons).

7. A. Ruge (dir.), Die deutsch-französischen Jahrbücher (1844), Reclam, 1977.

8. Voir E. Bloch, Les utopies sociales, in : Le principe espérance, II, Gallimard, 1982, p. 117-168; A.Eckhardt (dir.), Michail Bakunin - Konflikt mit Marx (2 vol.), éd. Karin Kramer, 2011.

9. F. Mignet, Histoire de la révolution française depuis 1789 jusqu'en 1814, Firmin Didot, Paris, 1924.

10. E. Gans, Storia della Rivoluzion francese / Die Geschichte der französischen Revolution, 1828, ed. bilingue, Rubbettino, 2011, pp.83-166. Merci à Alain Patrick Olivier d'avoir signalé cette source.

11. K.Marx, F.Engels, Le colonialisme, éd. critiques, 2018.

12. J. Derrida, Marx and sons, Galilée, 2020; voir sur ce sujet L. Sagradini, Livre phallus et oeil vaginal, Variations n.23, 2020.

13. K. Marx, Notes (années 1840), MEW, 2, p.43.

14. K. Marx, Misère de la philosophie / P.J. Proudhon, Philosophie de la misère, Union générale d'éditions 10/18, 1964.

15. K. Marx, Critique du programme de Gotha, ES, 2008.

16. K. Dietinger, Eine Leiche im Landwehrkanal. Die Ermordung Rosa Luxemburgs, Nautilus, 2018.

17. 208 cadres communistes allemands furent exécutés pendant la terreur stalinienne, dont au moins 7 membres de la direction du KPD: H. Weber, A. Herbst: Deutsche Kommunisten. Biographisches Handbuch 1918 bis 1945, Dietz, 2008.

18. H.R.Rabinowitz, Kosher Humor, Allia, 2008.

19. Voir la photo illustrant l'affiche des journées d'étude Le Floréal francfortois, Paris 8, juin 2021 : https://sens.univ-paris8.fr/IMG/pdf/journe_es_le_flore_al_francfortois_p8-2.pdf

20. S. Detlev, Das radikale Milieu, DUV, 2004.

21. G. Lukacs, Histoire et conscience de classe (sous-titre original: études dialectiques, 1922-23), éditions de Minuit, 1960; K. Korsch, Marxisme et philosophie (1920-23), éd. Allia, 2012.

22. Il s'agit d'un intellectuel qui est parvenu à infiltrer, pendant la seconde guerre mondiale, l'ambassade nazie au Japon, cela malgré la grande méfiance de Staline envers ce communiste conseilliste allemand. Sorge annonce la date exacte de l'attaque allemande contre l'Union soviétique, mais Staline méprise le message, puis Sorge annonce que le Japon n'attaquera pas par l'Est, ce qui permet le déplacement des troupes sibériennes et la défense de Moscou en 1941. Il fut décoré Héro de l'Union soviétique.

23. W. Benjamin, op.cit.

24. H.Marcuse, Le marxisme soviétique, Gallimard, 1958.

25. Marx, MEGA, 1, 1975.

26. K. Korsch, Marxisme et philosophie (1920-23), Allia, 2012.

27. Korsch, Marxismus und Philsophie, Offizin, 2017, p.117.

28. Si Althusser théorise une coupure ou rupture "épistémologique" (Pour Marx, Maspero, 1965), Kurz se contente plus tard d'écarter Feuerbach et la discussion critique avant 1848 de ses lectures marxiennes, et de faire abstraction de la lutte des classes : Kurz (dir.), Marx lesen. Die wichtigsten 
Texte von Karl Marx für das 21. Jahrhundert, Eichborn, 2001. Les travaux de la Théorie critique sont ignorés ici.

29. Korsch, op.cit., p.141.

30. Korsch, op.cit., p.202.

31. Lukacs, op.cit.

32. Voir p.ex. Adorno et alli, La personnalité autoritaire (1951), Allia, 2007.

33. H. Marcuse, Les vissicitudes de la dialectique in: Le marxisme soviétique, Gallimard, 1958, p.183-94.

34. Adorno, Dialectique négative, Payot, 2003.

35. Voir la traduction française publiée dans le présent n. 24 de la revue Variations, à partir du tapuscrit de Max Horkheimer, daté de 1931, rendu public en ligne par la section collections de la bibliothèque universitaire Johann Wolfgang Goethe: http://sammlungen.ub.uni-frankfurt.de/ horkheimer/content/pageview/6553861

36. Adorno, Minima moralia, Suhrkamp, 1959, p.284 (nous traduisons).

37. Adorno/Horkheimer, 'Zu Theorie und Praxis' in : Max Horkheimer, Gesammelte Schriften, Bd. 19, Fischer, 1996, S. 37-71 (nous traduisons les formulations citées).

38. A. Feenberg (2012), Le théâtre de l'absurde, traduction française dans le présent $\mathrm{n}$. de Variations n. 24. Dans ce commentaire, Feenberg se réfère à la position habermassienne: J. Habermas, $\mathrm{Ce}$ fascinant Monsieur Macron, L'Obs, 25 octobre 2017.

39. Dans un entretien radiophonique, Möglichkeiten der Utopie de 1964 (SWF) avec Bloch, Adorno tombe d'accord avec lui qu'un socialisme sans perspective utopique ne peut que se réifier sous la forme d'appareils.

40. Voir la note produite par la MEGA : Vol. 5, Manuskripte zur Deutschen Ideologie (1900 pages), De Gruyter, 2016.

41. Beiträge zur Marx-Engels Forschung, Sonderband 2, Argument, 2000.

42. Catalogue de la MEGA : mega.bbaw.de

43. R. Luxemburg, L'accumulation du Capital, Agone, 2019.

44. Merci à O. Negt et A.Demirovic de m'avoir renseigné.

45. Ch. Kirchoff et alli (dir.), Perspektiven einer neuen Marx-Lektüre. Festschrift für Hartmut Reichelt, éd. ça ira, 2000; A.Neumann, Marx après les marxismes, in Après Habermas, Delga, 2015, pp. 80-92; A.Neumann, Une mèche allumée, préface à J.M. Vincent, Fétichisme et société, éd. critiques, 2020, p.13.

46. La discussion part d'un texte de 1964, qui donne ce livre: A. Sohn-Rethel, Soziologische Theorie der Erkenntnis, Suhrkamp,1985.

47. H.G. Backhaus, Dialektik der Wertform, ça ira, 1998, J.M. Vincent, Critique du travail, PUF, 1987; J.M. Vincent, Un autre Marx. Après les marxismes, Page 2, 2001.

48. J.M. Vincent, Variations?, in : Variations n.1, Syllepse, 2001, p.8 : Variations entend bien réagir contre ceux qui jettent Marx aux orties, et avec lui les questions qu'il a posées. La revue prendre au contraire Marx au sérieux, suffisamment pour dialoguer avec lui en le critiquant, tout cela pour le prolonger au delà de ce qu'il a pu dire.

49. Adorno, Société industrielle ou capitalisme tardif? in : Société, Payot, 2012.

50. Adorno, Negative Dialektik, Suhrkamp, 1966 (nous traduisons).

51. P. Naville, De l'aliénation à la jouissance, Anthropos, 1959.

52. A.Einstein, Why socialism, Monthly review, NYC, 1er mai 1949.

53. L. Althusser, L'avenir dure longtemps, Stock, 1992; Althusser, Pour Marx, Maspero, 1965; Althusser et alli, Lire le Capital, Maspero, 1965.

54. L. Althusser, L'avenir dure longtemps, Stock poche, 1994, p.217-18: L'URSS "dut se résoudre à une négociation de compromis avec le Reich de Hitler. (...) Et qu'aurait-elle pu faire d'autre?". L'article que le dictionnaire du mouvement ouvrier consacre à Althusser le range parmi les intellectuels acquis au stalinisme, voir F. Matheron, Maitron, https://maitron.fr/spip.php? article9962 
55. E. Mandel, La formation de la pensée économique de Karl Marx, Maspero, 1967; J. Rancière, La leçon d'Althusser (1974), La Fabrique, 2012; L. Sève/L.Althusser, Correspondance, ES, 2018; E.P. Thompson, Misère de la théorie. Contre Althusser et le marxisme anti-humaniste, L'échappée, 2014; J.M. Vincent (dir.), Contre Althusser, 10/18, 1974, avec des contributions de Ch. Coliot Thélène, D. Avenat, A. Brossat, D. Bensaid et alli ; D. Bensäid (dir.), Contre Althusser - Pour Marx, La Passion, 1999; C. Castoriadis, Les crises d'Althusser. De la langue de bois à la langue de caoutchouc, Libre, N.4, 1978, pp. 239-54; O. Negt et A.Kluge, Geschichte und Eigensinn, Zweitausendeins, 1980.

56. Guy Debord, Correspondance : 1988-1994, Fayard, 2008.

57. Althusser, Actuel Marx n.67, PUF, 2020.

58. Althusser avait signalé Jacques Bidet comme le continuateur le plus prometteur des études marxiennes de ce groupe, voir J.Bidet, Que faire du Capital? Matériaux pour une refondation (1985), PUF, 2000.

59. Adorno, Minima moralia, Suhrkamp, 1959.

60. A.Neumann, III.1. Temps de travail nécessaire ou travail socialisé? in : Le salariat entre sphère privée, entreprise et espace public, thèse de doctorat, Paris V, 2005, pp.267-304; A. Neumann, Kritische Arbeitssoziologie, Schmetterling, 2010; A. Neumann, A brief History of Labour. From subsumption to mutltitude in : Joy Forver. The political economy of social creativity (dir. M. Kozlowski), MayFly Books, 2015, pp. 133-43.

61. Marx, Le chapitre VI (chapitre initialement inédit, 1867), ES, 2010.

62. Marx, Grundrisse, Dietz, 1979, p.185 (nous traduisons).

63. Kant, Streit der Fakultäten, 1798, deuxième partie, chap.6.; Kant, Le conflit des facultés (et autres textes sur la révolution), Payot, 2015.

64. R. Luxemburg (1917-18), La révolution russe, l'Aube, 2017.

65. O.Negt, L'espace public oppositionnel, Payot, 2007; H.J Krahl, Constitution et lutte des classes, entremonde, 2021.

66. A. Demirovic, Der nonkonformistische Intellektuelle, Suhrkamp, 1999, pp.881-951. D. Kraushaar, Frankfurter Schule und Studentenbewegung, documentation en trois volumes, HE, 1998.

67. Adorno, Gegen die Notstandsgesetze, in : Vermischte Schriften, I, stw, 1997, pp.396-97.

68. O. Negt, Achtundsechzig, Steidl, 1995;

69. A.Demirovic, op.cit., p.944.

70. H. Marcuse à Adorno, lettre du 12 janvier 1969, nous traduisons, voir archives Adorno et Benjamin, Berlin.

71. A. Demirovic, op.cit.

72. W. Gast, RAF erklärt sich zur Geschichte, tageszeitung, 22/4/1998.

73. P. Anderson, Sur le marxisme occidental, Maspero, 1976, p.149.

74. Le modèle initial est explicitement repris par R. Keucheyan, Hémisphère gauche. Une cartographie des théories critiques, (2010), La Découverte, 2017.

75. Nous parlons de créolisation au sens précis de E.Glissant, Le discours antillais, Gallimard, 1980, p.603 et 807 .

76. A. Davis, Femmes, race et classe (1980), Antoinette Fouque poche, 2020.

77. Arruz/Bhattacharia/Fraser, Manifeste féministe pour les 99\%, La Découverte, 2019.

78. J. Habermas, Auch eine Geschichte der Philosophie, Band 2, Suhrkamp, 2019.

79. J. Habermas, Ce fascinant Monsieur Macron, L'Obs, 25 octobre 2017.

80. A. Davis, discours au Teatro Baquedano, Chile, 2016 (nous traduisons).

81. G. Marcus, Lipstick traces. Une histoire secrète du 20ème siècle, Folio, 1998. 


\section{AUTEUR}

\section{ALEXANDER NEUMANN}

Professeur à Paris 8, LLCP, directeur de Variations 